\title{
Subtle and Personal Workspace Requirements for Visual Search Tasks on Public Displays
}

\author{
James R. Wallace \\ School of Public Health and \\ Health Systems \\ University of Waterloo \\ james.wallace@uwaterloo.ca
}

\author{
Ariel Weingarten \\ Cheriton School of Computer \\ Science \\ University of Waterloo \\ asweingarten@gmail.com
}

\author{
Edward Lank \\ Cheriton School of Computer \\ Science \\ University of Waterloo \\ lank@uwaterloo.ca
}

\begin{abstract}
We explore how users approach and define personal space on large, public displays. Our results show that users of public displays use one of two strategies for visual search tasks: minimizers create a small window and work up close to the display, and maximizers expand content to its full resolution and work at a distance. We show that these interaction styles match predicted 'personal' and 'subtle' interaction zones, characterize typical width and height requirements for these interactions, and show that these requirements are independent of the onscreen content's dimensions. Finally, we suggest practical guidelines for defining workspaces during personal and subtle interaction on large, public displays.
\end{abstract}

\section{ACM Classification Keywords}

H.5.2. Information Interfaces and Presentation (e.g. HCI): Interaction styles

\section{Author Keywords \\ Large Public Displays; Personal Workspace; Visual Search}

\section{INTRODUCTION}

As touch-interactive displays have become more affordable, larger, and more versatile their potential to move out of the office and support various short-lived, public interactions has been realized. Example interactions include looking up a colleague in an office directory, navigating a school campus with a provided map, or interacting with a museum exhibit. To support interactions with large displays, researchers have explored underlying theories of human behaviour such as proxemics and territoriality $[2,12]$, developed interaction techniques for multiple users and large displays [14], and observed the use of prototypes in public spaces [10].

However, much of this work has predated our ability to create and deploy large displays that extend beyond a few meters wide. Use cases for larger displays are markedly different. For example, in semi-public spaces, where users work as a

This is the author's version of the work. It is posted here by permission of ACM for your personal use. Not for redistribution. The definitive version was published in the Proceedings of the 2017 International Conference on Human Factors in Computing Systems on the ACM Digital Library http://dx.doi.org/10.1145/3025453.3025500 group, large displays have been envisioned as a 'canvas of infinite size' [6] where the entirety of a display is dedicated to supporting the group's work. Whereas in public settings, where users may approach a display to only briefly look up directions or enjoy a museum exhibit [8], it may be desirable to create a distinct, personal workspace for each individual.

While previous work has described 'ambient' displays that respond to approaching users $[13,16]$, there is little guidance in the literature towards how displays should respond to users in practice. For example, how large should a user's personal space be? And how does the content they are interacting with influence their requirements? Existing work has largely focused on developing support for proxemic interactions (e.g., $[2,5])$, but has not sought to understand how an individual's needs change with their proxemic relationship to the display. Our work addresses this gap in the literature.

We present an empirical study that explores how individuals define personal space and interact with content during a visual search task on a large display. We characterize typical width and height requirements for users working on a large display, and show that these requirements are independent of the on-screen content's aspect ratio. Our results also suggest that users of public displays tend towards one of two strategies when using a public display for visual search tasks: minimizers, who create a small window and manually search the image using touch inputs while standing near to the display (i.e., personal interactions), and maximizers who expand content to its full resolution and then step back to perform a hands-free search (i.e., subtle interactions). Finally, we suggest practical guidelines for the automatic creation of personal $(60 \times 60 \mathrm{~cm})$ and subtle $(100 \times 80 \mathrm{~cm})$ workspaces.

\section{INTERACTION WITH LARGE DISPLAYS IN PUBLIC}

A fundamental benefit of large displays is their ability to support interactions by multiple, simultaneous users. In public settings, these interactions are often short-lived, and may be performed by strangers on a single, shared surface. For example, Vogel and Balakrishnan [16] show how interactions with a display evolve from ambient, to implicit, to subtle, to personal in nature. Notably, in anticipating the shared use of public displays, Vogel and Balakrishnan write that these personal interactions "should be designed such that the disruption to the rest of the display is minimized, allowing simultaneous use by multiple people" (pg. 139). 

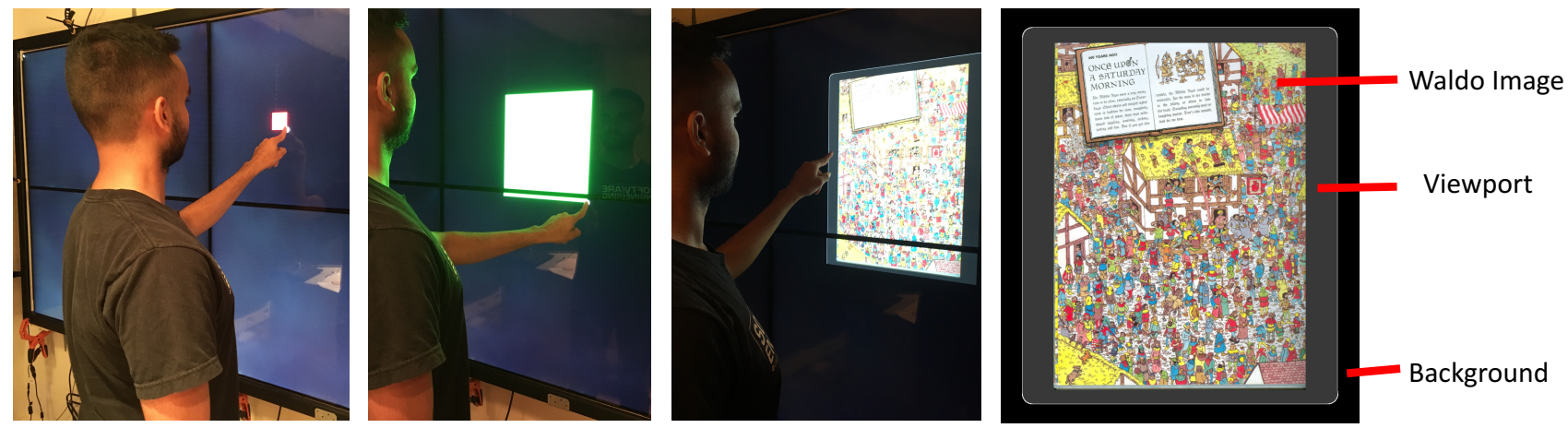

Figure 1. A participant in front of the $4 \times 1.2 \mathrm{~m}$ display. Viewports were initially created by drawing their diagonal with a single touch point. Viewports smaller than $400 \times 400 \mathrm{px}$ were not allowed, indicated by a red viewport on the display (Panel 1). Once the viewport exceeded this size is was displayed as green (Panel 2), at which point participants could release their touch to create an initial viewport (Panel 3). The contained Where's Waldo image was sized to maximize its area within the surrounding viewport (Panel 4).

To address this gap, research has carefully characterized space requirements around displays. Toney and Thomas [15] explored the 'reach envelope' over a tabletop display with an anthropometrically representative group of female users, and report that most interactions were constrained to a $12 \times 34$ $\mathrm{cm}$ region in front of a user. These regions are consistent with other work on tabletop displays (e.g., [1, 12]). While issues such as 'crowding' have frequently been identified as design considerations (e.g., $[11,12])$, specific guidance towards how a display should respond to an approaching user has not yet been explored in the literature. What research that does exist tends to be descriptive rather than prescriptive, such as Ryall et al.'s [11] suggestion that individuals like 'elbow room', and does not characterize how it should be provided in practice.

To address this shortcoming, we explore content management during a brief visual search task on a public display. Our results complement existing field work by characterizing interactions on a vertical display, and by carefully focusing on understanding how users may wish to manage on-screen content during short-lived interactions in a controlled setting.

\section{Content Management on Large Displays}

Public and ambient display research (e.g., $[13,16])$ often assumes that content will appear in front of a user as they approach a surface. On desktop systems, 'window management' is mature, with decades-old OS-level support to maximize and tile windows. However, public displays leverage novel, multitouch interaction metaphors such as Rotation aNd Translation (RNT) [9], are physically much larger than desktops, and are intended for short-lived interactions by non-experts. In these settings, display size is no longer a constraint, rather, interaction is constrained by human ergonomics, user preferences, and the on-screen content itself.

Further, we expect public displays to support various shortlived interactions, such as looking up a colleague in an office directory, navigating a school campus with a provided map, or interacting with a museum exhibit, with content that is often designed for use on a desktop display. On such large displays, 'maximizing' a portrait-oriented textual document has a drastically different effect than maximizing a landscape photograph. For example, on the display used in our study portrait-oriented content maximized to fill the display would have a width of $68 \mathrm{~cm}$, nearby five times the width of the reach profiles observed by Toney et al. [15], whereas maximized landscape-oriented content would have a width of $213 \mathrm{~cm}-$ extending beyond a typical user's reach.

Our research characterizes how users manage content during short-lived visual search tasks, such as approaching a public display to check for directions. In particular, we show that for such tasks, the aspect ratio of on-screen content plays a small role in determining a user's workspace needs, and that users tend to either minimize content and navigate through touch, or maximize content and stand away from the display while performing a visual search.

\section{STUDY}

To explore these questions, we invited participants to interact with a public display. By conducting our study under controlled circumstances we were able to carefully measure interactions with the display. We chose to vary the aspect ratio of on-screen content, e.g. portrait vs landscape, to explore how an individual's space requirements change as they work with different content. Notably, we did not control how or where participants interacted with the large display, and instead simulated conditions in which users might approach and briefly interact with such a display in public.

\section{Participants}

21 Science, Technology, Engineering, and Mathematics students ( 4 women) between the ages of 18 and $23(\mu=20.3)$ were recruited from a local university, and received a candy bar for their participation.

\section{Experimental Setup}

Participants completed trials on a 170" display measuring 4.1 $\times 1.2 \mathrm{~m}$. The display was composed of $8 \times 46 "$ display panels arranged in a $4 \times 2$ grid, for a total resolution of $7680 \times$ 2160 pixels (16MP at $47 \mathrm{PPI})$. The display supported 32 touch points via a PQ Labs infrared touch frame (Figure 1).

\section{Experimental Task and Design}

Our study utilized a $3 \times$ ASPECT RATIO between-subjects design, where participants performed a short visual search task in 
one of three aspect ratios: landscape (16:9), square (1:1), and portrait (9:16). The experimental task consisted of searching a single "Where's Waldo" image for the eponymous character and was chosen due to its similarity to real-world, transient tasks such as stopping to consult a kiosk for directions.

Participants first stepped up to the large display and created a viewport by dragging their finger to define its diagonal dimension; recorded as INITIAL VIEWPORT size. The Where's Waldo image was then displayed within the viewport, with a size calculated to maximize its area without cropping or magnification beyond its native resolution. During pilot testing, we found that our touch frame occasionally detected false touch events, creating unintentionally small viewports, and thus our final design only allowed viewports exceeding $400 \times$ $400 \mathrm{px}$. After the image appeared, participants were instructed to adjust the viewport and image for comfort before beginning their visual search. Adjustments to the viewport were recorded as WORKSPACE ADJUSTMENTS.

The viewport continued to be adjustable throughout the task. While searching for Waldo, the interface allowed users to translate the image within the viewport using a single touch point and scale the Waldo image using a two-finger pinch-tozoom metaphor. The viewport could also be manipulated by the same techniques. When part of the image fell outside of the viewport's boundary, the image was cropped such that only the portion within the viewport's bounds was rendered. To complete the task, participants informed the investigator of Waldo's location and two other objects verbally.

\section{Procedure}

Upon arriving, participants completed an informed consent form and brief demographic questionnaire. They were then provided an introduction to the touch screen and experimental software and given the opportunity to ask any clarifying questions before starting the experimental trials. In particular, participants were informed that the display was intended to be used in public, and that they should interact with the experimental software as if they were walking up to a display in public space. Participants then completed a single experimental trial, where they placed a viewport and "found Waldo" and two other items in the puzzle. After finding Waldo, participants were prompted to reflect on whether the viewport was optimally sized for their task and then thanked for their time. In total, each session lasted approximately 5 minutes.

\section{Data Collection and Analysis}

The time taken to complete each trial, initial placement of personal workspaces, and participant interactions with experimental software were logged. We analyzed interaction data to determine the initial size of personal workspaces, as well as any adjustments made through the course of completing the task. To analyze the collected data we performed analysis of variance (ANOVA) tests with an alpha of 0.05 over dependent variables including the width and height of participants' viewports, the aspect ratio of viewports, where aspect ratio is defined as the width of an on-screen window divided by its height. Thus, square viewports (1:1) would have an as-

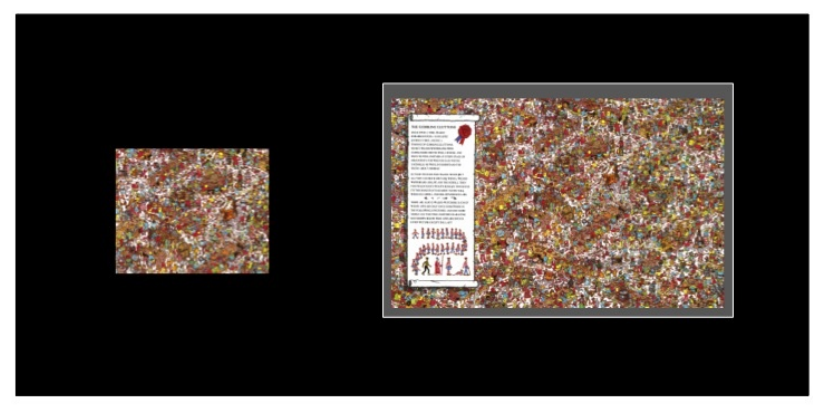

Figure 2. Participants fell into one of two categories: Minimizers (left) who created a small viewport, requiring translation to view the entire content image, and Maximizers (right) who created a viewport large enough to view the image at the participant-set resolution

pect ratio of 1 , portrait viewports (9:16) 0.56 , and landscape viewports would have an aspect ratio of 1.78 (16:9).

\section{Results}

We report on two aspects of territoriality as observed in the study: how participants approach and initially create personal workspaces, and how the workspace was used once established on the display.

\section{Defining a Workspace}

On average, participants created an initial viewport that measured $65 \mathrm{~cm}$ wide $(\sigma=34.4) \times 54 \mathrm{~cm}$ high $(\sigma=22.3)$. For reference, individual panels within the large display measured $100 \mathrm{~cm}$ wide $\times 58 \mathrm{~cm}$ high. Our analyses revealed no differences in window height $\left(F_{2,18}=1.677, p=0.228, \eta^{2}=\right.$ $0.218)$ or width $\left(F_{2,18}=1.524, p=0.257, \eta^{2}=0.203\right)$ between content ASPECT RATIO conditions.

Our analysis revealed no differences between the defined viewport's aspect ratio across the three content ASPECT RATIO conditions $\left(F_{2,18}=0.032, p=0.968, \eta^{2}=0.005\right)$, and indicated that the different conditions accounted for less than $1 \%$ of the variance encountered in our model. Across all participants, viewports were initially defined with an average aspect ratio of $1.158(\sigma=0.093)$.

\section{Workspace Adjustments}

Throughout the trials, participants often resized the viewport. At the end of trials viewports measured on average $88 \mathrm{~cm}(\sigma=$ $43.2)$ wide $\times 74 \mathrm{~cm}(\sigma=25.8)$ high, corresponding to a $35 \%$ increase in each dimension and a $80 \%$ increase in the viewport's total area. Our analysis revealed significant differences for both the final width $\left(F_{2,18}=3.885, p=.050, \eta^{2}=.393\right)$ and height $\left(F_{2,18}=6.357, p=.013, \eta^{2}=.514\right)$ of viewports, where viewports for portrait content were larger than those for landscape or square content; a result of its needing to scale more drastically to conform to square or rectangular content.

Post hoc analysis of final viewport dimensions revealed that participants fell into one of two groups: maximizers (10/21 participants), who sized their viewport to be larger than the Waldo image and minimizers (11/21 participants), who created a viewport that was smaller than its Waldo image content (Figure 2). Participants identified as minimizers created a viewport with an initial size of $53 \times 49 \mathrm{~cm}$ on average, and 
made adjustments of less than $8 \mathrm{~cm}$ for the viewport's width and height. 8/11 minimizers made no adjustments to their viewport's size after its initial placement. On the other hand, maximizers created a viewport with an average initial size of $80 \times 63 \mathrm{~cm}$ and made larger adjustments averaging $19 \mathrm{~cm}$ in each direction. Only $2 / 10$ of the maximizers did not resize their viewport.

\section{DISCUSSION}

Our findings suggest that ASPECT RATIO plays a small role in determining workspace requirements on public displays. However, we also observed that participants fell into one of two categories: minimizers or maximizers. Minimizers (11/21) created a small workspace which they then searched by panning within the viewport. On the other hand, maximizers $(10 / 21)$ created a viewport large enough to view the entire image, and stepped back from the display to perform the search task. Interestingly, these interaction styles correspond to those described by Vogel and Balakrishnan [16] as taking place in 'personal' and 'subtle' spaces, respectively, and our participants were evenly divided between the two categories, indicating a need to provide support for both groups. We now discuss how to provide appropriate support for these interaction styles.

\section{Supporting Minimizers and Personal Interaction}

The majority of participants identified as minimizers (8/11) created a small viewport on the large display, and made no further adjustments to its size as they performed the visual search task. For these users, the native size of on-screen content appeared to play only a small role in defining a viewport, and they preferred to adopt a 'pan and scan' search strategy. Our measurements suggest a $60 \times 60 \mathrm{~cm}$ workspace was sufficient for their needs.

Interestingly, for minimizers this square workspace appears to be consistently useful. For example, one might expect that users would create viewports that conform to their content's dimensions, or, given the widespread use of 16:9 displays prefer a landscape workspace. However, our analysis of effect size suggests that participants disregarded the aspect ratio of on-screen content $\left(\eta^{2}=0.005\right)$. The average viewport defined by participants was more square than landscape, which we interpret as being driven by human ergonomics (i.e., reach and field of view).

This consistency suggests that a $60 \times 60$ workspace may serve as a practical rule of thumb for up-close, 'personal' interactions on wall displays. Notably, this guidance differs from that available for digital tabletop interaction. Our observed workspace dimensions of $65 \times 54 \mathrm{~cm}$ are approximately $7 \times$ larger than the $12 \times 34 \mathrm{~cm}$ region observed by Toney et al. [15] on digital tabletops. These differences may arise because vertical displays afford opportunities for interaction across a greater area, or 'reach envelope', and content is more easily viewed on vertical displays from a distance than on tabletop displays. Therefore, we suggest that care should be taken in developing 'write once, run everywhere' software for multitouch surfaces, and that form factor is an important design consideration for interface design, such as in determining appropriate menu placement.

\section{Supporting Maximizers and Subtle Interaction}

The remainder of our participants (10/21) chose to perform the visual search task a few steps away from the display, in the 'subtle' interaction zone. Our observations suggest that for these users a larger on-screen area is more appropriate, averaging $100 \times 80 \mathrm{~cm}$ or approximately twice the area of 'personal' viewports. These viewports were sufficiently large to view the entire image, without a need to 'pan and scan'.

Further, our results suggest a practical compromise between presenting content that is large enough to support subtle engagement, while also preserving screen space for new users. For example, it may be tempting to maximize the screen size of content for these users, however, doing so also limits the amount of screen space available to other, nearby users, particularly for landscape content. Instead, our results suggest that a simple rule of thumb is to size content during subtle interactions at approximately twice the area of that used for personal interactions. This rule of thumb aims to strike a balance between individual preference and supporting interaction by multiple, independent users.

\section{LIMITATIONS}

We set out to explore short-lived visual search tasks on public displays. Our findings help to characterize on-screen space requirements in these settings, however, some caution should be exercised when interpreting these results in other contexts. For example, we intentionally studied transient interactions by single users, and our results may not apply to work longer in duration or for extended collaborative work around a large display. Work at CHI suggests that users adjust their use of gestures [7] and interpersonal space [17] in these settings. Similarly the presence of interior bezels, may influence user behaviour on and around large public displays. For example, while research suggests that bezels have little impact on visual search performance [3,18], they may impact where users stand or choose to place content [4].

\section{CONCLUSION}

Large displays are increasingly available for short-lived interactions in public; our research provides practical guidance towards how to create personal and subtle workspaces on public displays. We conducted an empirical study that characterizes workspace needs for a visual search task, where participants stood both up-close to a display (personal interaction) and for those at a distance (subtle interaction). In reflecting on our results we have shown that 1) content properties such as aspect ratio play a minimal role in determining on-screen space requirements, and 2) that users fall into two groups: 'minimizers' and 'maximizers', corresponding to personal and subtle zones of interaction, respectively. Our results provide insight into the on-screen space requirements for users interacting in these zones, and into how space can be appropriately partitioned to strike a balance between an individual's needs, and the need to support multiple users as they approach a display.

\section{ACKNOWLEDGMENTS}

This research was funded by the Natural Sciences and Engineering Research Council of Canada. 


\section{REFERENCES}

1. Trent Apted, Judy Kay, and Aaron Quigley. 2006. Tabletop Sharing of Digital Photographs for the Elderly. In Proceedings of the SIGCHI Conference on Human Factors in Computing Systems (CHI '06). ACM, New York, NY, USA, 781-790. DOI :

http://dx. doi . org/10.1145/1124772.1124887

2. Till Ballendat, Nicolai Marquardt, and Saul Greenberg. 2010. Proxemic Interaction: Designing for a Proximity and Orientation-aware Environment. In Proc. ITS 2010. ACM, New York, NY, USA, 121-130. DOI :

http://dx . doi .org/10.1145/1936652 . 1936676

3. Xiaojun Bi, Seok-Hyung Bae, and Ravin Balakrishnan. 2010. Effects of Interior Bezels of Tiled-monitor Large Displays on Visual Search, Tunnel Steering, and Target Selection. In Proceedings of the SIGCHI Conference on Human Factors in Computing Systems (CHI '10). ACM, New York, NY, USA, 65-74. DOI :

http://dx.doi.org/10.1145/1753326.1753337

4. Xiaojun Bi and Ravin Balakrishnan. 2009. Comparing Usage of a Large High-resolution Display to Single or Dual Desktop Displays for Daily Work. In Proceedings of the SIGCHI Conference on Human Factors in Computing Systems (CHI '09). ACM, New York, NY, USA, 1005-1014. DOI :

http : //dx . doi .org/10.1145/1518701.1518855

5. Saul Greenberg, Sebastian Boring, Jo Vermeulen, and Jakub Dostal. 2014. Dark Patterns in Proxemic Interactions: A Critical Perspective. In Proceedings of the 2014 Conference on Designing Interactive Systems (DIS '14). ACM, New York, NY, USA, 523-532. DOI : http: //dx. doi . org/10 . 1145/2598510.2598541

6. Michael Haller, Jakob Leitner, Thomas Seifried, James R. Wallace, Stacey D. Scott, Christoph Richter, Peter Brandl, Adam Gokcezade, and Seth Hunter. 2010. The NiCE Discussion Room: Integrating Paper and Digital Media to Support Co-Located Group Meetings. In Proceedings of the SIGCHI Conference on Human Factors in Computing Systems (CHI '10). ACM, New York, NY, USA, 609-618. DOI:http://dx.doi.org/10.1145/1753326.1753418

7. Uta Hinrichs and Sheelagh Carpendale. 2011. Gestures in the Wild: Studying Multi-touch Gesture Sequences on Interactive Tabletop Exhibits. In Proceedings of the SIGCHI Conference on Human Factors in Computing Systems (CHI'11). ACM, New York, NY, USA, 3023-3032. DOI :

http: //dx.doi .org/10.1145/1978942 . 1979391

8. Daniel Klinkhammer, Markus Nitsche, Marcus Specht, and Harald Reiterer. 2011. Adaptive Personal Territories for Co-located Tabletop Interaction in a Museum Setting. In Proceedings of the ACM International Conference on Interactive Tabletops and Surfaces (ITS '11). ACM, New York, NY, USA, 107-110. DOI :

http : //dx . doi . org/10 . 1145/2076354.2076375

9. Russell Kruger, Sheelagh Carpendale, Stacey D. Scott, and Anthony Tang. 2005. Fluid Integration of Rotation and Translation. In Proceedings of the SIGCHI
Conference on Human Factors in Computing Systems (CHI '05). ACM, New York, NY, USA, 601-610. DOI : http://dx.doi . org/10.1145/1054972.1055055

10. Peter Peltonen, Esko Kurvinen, Antti Salovaara, Giulio Jacucci, Tommi Ilmonen, John Evans, Antti Oulasvirta, and Petri Saarikko. 2008. It's Mine, Don't Touch!: interactions at a large multi-touch display in a city centre. In Proc CHI. ACM, New York, NY, USA, 1285-1294. DOI:http://dx.doi .org/10.1145/1357054.1357255

11. Kathy Ryall, Meredith Ringel Morris, Katherine Everitt, Clifton Forlines, and Chia Shen. 2006. Experiences with and observations of direct-touch tabletops. In Proceedings Tabletop 2006. IEEE, 8-pp.

12. Stacey D. Scott, M. Sheelagh T. Carpendale, and Kori M. Inkpen. 2004. Territoriality in Collaborative Tabletop Workspaces. In Proceedings of the 2004 ACM Conference on Computer Supported Cooperative Work (CSCW '04). ACM, New York, NY, USA, 294-303. DOI : http://dx.doi.org/10.1145/1031607.1031655

13. Norbert A Streitz, Carsten Röcker, Thorsten Prante, Richard Stenzel, and Daniel van Alphen. 2003. Situated interaction with ambient information: Facilitating awareness and communication in ubiquitous work environments. In HCI International 2003.

14. Anthony Tang, Mattias Finke, Michael Blackstock, Rock Leung, Meghan Deutscher, and Rodger Lea. 2008. Designing for Bystanders: Reflections on Building a Public Digital Forum. In Proceedings of the SIGCHI Conference on Human Factors in Computing Systems (CHI '08). ACM, New York, NY, USA, 879-882. DOI : http://dx. doi . org/10.1145/1357054.1357193

15. Aaron Toney and Bruce H. Thomas. 2006. Applying Reach in Direct Manipulation User Interfaces. In Proceedings of the 18th Australia Conference on Computer-Human Interaction: Design: Activities, Artefacts and Environments (OZCHI '06). ACM, New York, NY, USA, 393-396. DOI : http://dx.doi .org/10.1145/1228175.1228255

16. Daniel Vogel and Ravin Balakrishnan. 2004. Interactive public ambient displays: transitioning from implicit to explicit, public to personal, interaction with multiple users. In Proc UIST '04. ACM, 137-146. DOI : http://dx.doi . org/10.1145/1029632.1029656

17. James R. Wallace, Nancy Iskander, and Edward Lank. 2016. Creating Your Bubble: Personal Space On and Around Large Public Displays. In Proceedings of the 2016 CHI Conference on Human Factors in Computing Systems (CHI'16). ACM, New York, NY, USA, 2087-2092. DOI:

http: //dx . doi .org/10.1145/2858036.2858118

18. James R. Wallace, Daniel Vogel, and Edward Lank. 2014. Effect of Bezel Presence and Width on Visual Search. In Proceedings of The International Symposium on Pervasive Displays (PerDis '14). ACM, New York, NY, USA, Article 118, 6 pages. DOI:

http://dx.doi .org/10.1145/2611009.2611019 